\section{Crystalline sponges to aid X-ray analysis}

\section{By Tracey Baas, Senior Editor}

Researchers from The University of Tokyo have used porous metal complexes dubbed crystalline sponges to enable X-ray crystallography of natural small molecules that do not readily crystallize. ${ }^{1}$ The group is forming a company to extend the method to large peptides and proteins.

$\mathrm{X}$-ray single-crystal diffraction is the only method that provides direct structural information at the atomic level of a compound, and it is considered more reliable than NMR or mass spectrometry. However, molecules of interest must be obtained as single crystals, which is not always possible.

Determining a crystallization protocol is a time-consuming, trial-anderror procedure that cannot be automated. Each molecule requires different crystallization conditions, and often those conditions are hard or impossible to determine.

To overcome these limitations, the Tokyo team turned to crystalline sponges it has developed over the last two decades. The sponges provide a crystalline framework that can host small volumes of guest compounds in an orderly disposition.

A crystalline sponge with a volume of about $100 \mu \mathrm{m}^{3}$ can contain about $5 \mu \mathrm{g}$ of target molecule from a drop of liquid or when immersed in a small liquid sample.

The pores can accommodate differently shaped molecules and are able to hold trapped molecules in one orientation. The sponges loosely confine the molecules, allowing for reversible binding, which results in the molecules going through several different binding orientations before finding the one with the lowest energy. ${ }^{2}$

Because most molecules tend to adopt their lowest-energy conformation, the result is large numbers of molecules held in the same regular, repeated arrangement, which then is detectable with X-ray analysis.

As proof of concept, the researchers set out to determine the structures of eight small molecules constrained in sponges. Five of the structures were readily determined-three were incorrectly solved because of common problems with crystallographic analysis such as atom misassignment, symmetry problems and adsorbed molecule disorder, but these issues were overcome by combining the X-ray analysis with mass spectrometry data.

Importantly, the technique solved the structures of a variety of different molecular compounds, including polycyclic, nonaromatic and nonplanar molecules.

The Tokyo team then went on to analyze polymethoxyflavones extracted from orange peel. Following analytical high-performance liquid chromatography separation, which provided a few micrograms of each of the three main flavonoid components, each of the components was put into a crystalline sponge and their X-ray crystal structures solved.

Finally, the researchers showed that the technique could be used to determine the structure of chiral compounds, which entails determining the absolute configuration of their chiral centers. They solved the structure and stereochemistry of the anthelminthic drug santonin, which has four chiral centers, and that of the marine sponge natural product miyakosyne A, which has three chiral centers.

Results were published in Nature.

Yurij Mozharivskyj, associate professor of chemistry at McMaster University, wanted to see the technique further validated using pharmaceutical compounds and metabolites, specifically those with chiral centers that are difficult to crystallize. He was also curious to see if the technique could be extended for use with peptides or proteins.

"So far, the molecules were relatively simple and had a limited number of primary attachment points to the host lattice. With peptides or proteins, there can be a large number of equivalent attachment points and thus a number of possible configurations in the host structure," he said.

Jon Clardy, professor of biological chemistry and molecular pharmacology at Harvard Medical School and co-director of the Infectious Disease Program at the Broad Institute of MIT and Harvard, agreed. "I think various alternatives for the metal complexes that make up the sponges will have to be investigated. In this work, the researchers focused on two different crystalline sponges. The current sponges are much too small to accommodate proteins. The upper limit on the cavity size of the crystalline sponges will have to be determined experimentally."

According to Yasuhide Inokuma, the team has already "used sponges with different metal-complex composition to solve the structure "In this work, the
researchers focused on
two different crystalline
sponges. The current
sponges are much too small
to accommodate proteins.
The upper limit on the
cavity size of the crystalline
sponges will have to be
determined experimentally."
$\quad$ - Jon Clardy,
Harvard Medical School of a polycyclic hydrocarbon with a molecular weight of over 500 daltons." Inokuma, lead author on the paper, is assistant professor of applied chemistry at the University of Tokyo. "We are confident that by developing sponges with the correct pore size, our strategy will also be applicable to solve the structures of peptides and proteins."

Inokuma added that the group "is forming a company to provide the crystalline sponges in addition to X-ray analysis of samples requested by academic and company laboratories."

The University of Tokyo has applied for patents covering the synthetic procedures used to generate the crystalline sponges and the methods for loading trace amounts of samples into the sponges.

The X-ray analytical instrumentation company Rigaku Corp. has nonexclusively licensed the use of the techniques, and the Tokyo team is in discussions with pharmaceutical companies about licensing the technique to analyze in-house compounds.

Baas, T. SciBX 6(15); doi:10.1038/scibx.2013.355

Published online April 18, 2013 


\section{REFERENCES}

1. Inokuma, Y. et al. Nature; published online March 27, 2013; doi:10.1038/nature11990

Contact: Makoto Fujita, The University of Tokyo, Tokyo, Japan e-mail: mfujita@appchem.t.u-tokyo.ac.jp

2. Stallforth, P. \& Clardy, J. Nature 495, 456-457 (2013)
COMPANIES AND INSTITUTIONS MENTIONED

Broad Institute of MIT and Harvard, Cambridge, Mass.

Harvard Medical School, Cambridge, Mass.

McMaster University, Hamilton, Ontario, Canada

Rigaku Corp., Tokyo, Japan

The University of Tokyo, Tokyo, Japan 